\title{
Non-Invasive Ventilation in COVID-19-Related Respiratory Failure
}

\author{
Irmi Syafa'ah ${ }^{*}$ (iD), Young-Jae Cho ${ }^{2}$ iD \\ ${ }^{1}$ Department of Pulmonology and Respiratory Medicine, Faculty of Medicine, Universitas Airlangga, Surabaya, Indonesia. \\ ${ }^{2}$ Division of Pulmonary and Critical Care Medicine, Department of Internal Medicine, Seoul National University College of \\ Medicine, Seoul, South Korea.
}

\section{ARTICLE INFO}

Article history:

Received 15 July 2021

Received in revised form

26 September 2021

Accepted 28 September 2021

Available online 30 September 2021

Keywords:

COVID-19,

Infectious disease,

Mechanical ventilation,

Non-invasive ventilation.

\begin{abstract}
Non-invasive ventilation (NIV) provides mechanical ventilation that does not require definitive airway clearance using an endotracheal tube or tracheostomy. Since its early development in the 1980s, the use of NIV has become increasingly popular in the last three decades. However, its usage on COVID-19-related respiratory failure still lacks guidelines, although several recent studies have shown its benefits. Many aspects, ranging from indications or patient selection, timing to start, understanding the predictor factors of failure, and choosing suitable interfaces, are keys of success for NIV. In principle, each patient has a different condition and should be treated case by case. NIV is not an absolute solution, and intubation can still be the first choice if NIV is deemed less beneficial for the patient.
\end{abstract}

\section{INTRODUCTION}

Since December 2019, the world seems forced to adapt to a new disease called coronavirus-19 (COVID19) caused by the SARS-CoV-2 virus. Starting from Wuhan, China, the disease has become a pandemic that has infected more than 200 million people, with a death rate of more than 4.6 million people worldwide. As of 14 September 2021, the number of confirmed COVID19 cases in Indonesia reached 4.1 million cases and more than 140,000 deaths. ${ }^{1}$

Respiratory failure is a complication and the leading cause of death in COVID-19 patients due to pulmonary dysfunction resulting in hypoxemia, called COVID-19-associated acute respiratory distress syndrome (CARDS). ${ }^{2,3}$ Various theories have emerged about the underlying mechanism and are considered 'atypical,' with pretty different characteristics from ARDS. ${ }^{2,4}$ The management of respiratory failure is challenging for clinicians who work inside and outside critical care due to its heterogeneous symptoms and high mortality. ${ }^{5}$
Initially, this condition required mechanical ventilation, where early intubation and invasive ventilation were considered more effective than noninvasive ventilation (NIV). However, there are ongoing debates around the risks-benefits since good evidence is still lacking. ${ }^{6}$ Some experts believe early intubation may avoid self-induced lung injury ${ }^{7}$, while those who were against were considering the high number of COVID19-related deaths that may be worsened by ventilatorinduced lung injury. ${ }^{3}$ However, as the pandemic worsens, the numbers of critically ill patients requiring mechanical ventilation exceed the ventilator's availability. Even for intubated patients, ventilation weaning is challenging and not always successful resulting in extended care outside the intensive care unit (ICU) for those in 'queue' for ventilators. ${ }^{6,8}$

As a result, NIV, mostly used outside the ICU, was in high demand to solve the problems. Growing evidence has shown that NIV, including Continuous Positive Airway Pressure (CPAP) and Bi-Level Positive

*Corresponding author: irmi-syafaah@fk.unair.ac.id 
Airway Pressure (BiPAP), may have some benefit than initially thought. ${ }^{9,10}$ NIV may help patients in the early stages by preventing deterioration and further reduce the need for intubation. Furthermore, they can be used outside the ICU that allows the physician to treat a large number of patients but with the risk of staff contamination. Still, its usage among COVID-19 patients lack in the guideline to accurately determine patients outcome. Perfect timing and proper patients selection are the keys for successful use of NIV. 6,8

\section{COVID-19 Associated Acute Respiratory Distress Syndrome (CARDS)}

Knowledge about what happened in respiratory failure due to COVID-19 is necessary to give better respiratory management to the patients. Most COVID19 related respiratory failures fall into ARDS. COVID19 ARDS (CARDS) is the leading cause of respiratory failure in COVID-19 patients, but not all cases of acute respiratory failure in COVID-19 patients are associated with ARDS. ${ }^{2}$ Gattinoni, et al. stated that pneumonia COVID-19, although included in most conditions according to the Berlin ARDS definition ${ }^{11}$, is a specific disease characterized by severe hypoxemia, which is often associated with near-normal respiratory system compliance. The combination of these two things is rarely found in severe ARDS, thus the term CARDS appears as a differentiator, namely ARDS caused by COVID-19. 2,7,12

COVID-19 patients with severe hypoxemia, despite sharing a single etiology (SARS-CoV-2), can present with very different conditions. The patient may appear normal ("silent" hypoxemic) or very breathless; moderately responsive to nitric oxide or not; very hypocapnic or normo/hypercapnic; and between responsive to the prone position or not. Therefore, the fact that the same disease can give a very diverse clinical picture further increases the effort to deal with this case. $^{13}$

\section{COVID-19 Pneumonia, L Type}

Initially, pneumonia in COVID-19 presented an L phenotype with the following characteristics ${ }^{13}$ :

- Low elasticity: lung compliance that is close to normal indicates that the amount of gas in the lungs is also close to normal.

- Low ventilation-to-perfusion (VA/Q ratio) ratio: because the amount of gas in the lungs is close to normal, hypoxemia is more accurately described as a loss of perfusion regulation and hypoxic vasoconstriction. Thus, at this stage, the pulmonary artery pressure should also be close to normal.

- Low lung weight: only ground-glass density is seen on CT scans, especially in the subpleural and along the pulmonary fissures. Thus, lung weight only increases slightly.

- Low recruitment ability: the number of nonaerated/non-aerated lungs is very low, thus there is no significant area that can be recruited.

The keys to ventilation management are to aim at optimal lung-protective ventilation and avoid patient self-inflicted lung injury (P-SILI). ${ }^{4}$

\section{COVID-19 Pneumonia, H Type}

This type of $\mathrm{H}$ pattern follows the criteria for severe ARDS, namely hypoxemia, bilateral infiltrates, decreased compliance, increased new weight, and potential recruits. Figure 5A shows a change in the CT scan of a type $\mathrm{L}$ patient on admission, and his transition to type $\mathrm{H}$ after seven days of noninvasive support. ${ }^{13}$

Type H COVID-19 pneumonia with the following characteristics: ${ }^{13}$

- High elasticity: decreased lung gas volume due to increased edema will also increase lung elasticity.

- High right-to-left shunt: this is due to the fraction of cardiac output that flows into the unaerated tissue that appears in the area of the lung that needs it due to increased edema and superimposed pressure.

- High lung weight: quantitative analysis of CT scans showed a significant increase in lung weight $(>1.5 \mathrm{~kg})$ as a result of the severity of ARDS.

- High recruitment ability: increased number of nonaerated/non-aerated lungs, with increased recruitment.

NIV refers to the technique of providing mechanical ventilation that does not require definitive airway clearance using an endotracheal tube or tracheostomy. Its use with positive pressure ventilation increased rapidly in recent years through nasal aids and face masks, which are thought to be able to provide better airway protection. ${ }^{16,17}$ NIV that provides positive pressure is better known as non-invasive positive pressure ventilation (NPPV). ${ }^{14}$ 
With the concept of phenotype in CARDS, of course, the respiratory management that can be given to type $\mathrm{L}$ and type $\mathrm{H}$ will be different. While type $\mathrm{H}$ patients should be treated as severe ARDS, for the type L patients with dyspnea, several NPPV options are available, such as CPAP or BiPAP. High-flow nasal oxygen (HFNO) is also one non-invasive option that may help but differs in the positive pressure provided. In this emergency condition, efforts to free the airway are the main thing that needs to be done. Selection of the right type and oxygen therapy aids can also affect the patient's recovery. ${ }^{15}$

NIV surely is not enough to handle all severe COVID-19 patients, as shown by recent evidence. ${ }^{6}$ The indications for the use of NIV are broadly (1) to prevent impending (not yet occurring) acute or post-extubation failure, (2) in the early phase, when respiratory failure has occurred, to prevent the need for endotracheal intubation, and (3) as an alternative to invasive ventilation in the more severe stages of acute respiratory failure or to facilitate the weaning process of mechanical ventilation, as in most COVID-19 patients. ${ }^{14,16}$ Despite its benefit in providing better oxygenation and reduce work of breathing, NIV may not stop the progressions of the disease, let alone replace invasive ventilation. ${ }^{17}$

\section{Continuous Positive Airway Pressure (CPAP)}

One method of choice for severe COVID-19 patients is CPAP. It delivers a single continuous pressure, measured in $\mathrm{cmH}_{2} \mathrm{O}$, and maintained throughout the respiratory cycle, during inspiration and expiration. CPAP does not work like NIV which actively supports respiratory muscles, or assist with the delivery of tidal volume $\left(\mathrm{V}_{\mathrm{T}}\right)$. Still, it maintains Positive End Expiratory Pressure (PEEP), which could decrease atelectasis by preventing alveolar collapse, increasing the alveolus's surface area, and improving V/Q matching, resulting in improved oxygenation. ${ }^{18,19}$

CPAP was said as the primary mode of nonrespiratory support for hypoxemic COVID-19 patients. The initial setting was suggested at $10 \mathrm{cmH}_{2} \mathrm{O}$ and $60 \%$ of oxygen. The target oxygenation was $\mathrm{SpO}_{2}$ of 945 to $96 \%$ or $88 \%$ to $92 \%$ for acute or acute on chronic type II respiratory failure. The key to the successful use of CPAP is patient's tolerance. It means patients selection is highly contributed to the outcome. ${ }^{20}$

A sealed system is one requirement that needs to be fulfilled for CPAP to be effective, which is mostly applied using a tight-fitting mask or even helmet. Both have their benefits and disadvantages. ${ }^{6,20}$ Tight-fit masks are the most familiar interface in this pandemic since its relatively easy-to-acquire compared to others, but prolonged use potentially causes pressure damage in the nasal bridge, which may lead to ulcers. Tight-fit application is very important in oxygen delivery since an ill-fitting mask leads to significant leaks resulting in poor inflations of the lung, and the dry leakage air will cause irritation, abrasion, even edema to eye tissue. ${ }^{6}$ The usage of this interface has been either reduced or restricted to an isolation room in some areas due to potential airborne spreading. ${ }^{21,22}$

Meanwhile, helmet CPAP (H-CPAP) has been recommended by European guidelines as one of COVID-19 related respiratory failure management strategies since it may reduce the aerosolization and exposure to SARS-CoV-2 compared to HFNO and face mask (FM). ${ }^{21,23}$ H-CPAP also allows enteral nutrition and hydration, has limited air leaks, better tolerability, and less risk for facial pressure. Both face masks and helmets might be used outside the ICU, but the helmet is bigger and might be uncomfortable for some patients. Moreover, the noise generated from the high flow required impedes communication with staff and, for some, causes claustrophobia that may worsen the patient's condition. . $^{21,24}$

Both interfaces have some risks, including gastric distention due to air swallowing that may lead to aspiration of gastric content. In COVID-19 patients whose lungs are less compliant, the risk of barotrauma should be observed regularly since it may lead to cardiac arrest. $^{20}$ Chiumello, et al. demonstrated the equal performance of these two interfaces for improving inspiratory effort and work of breathing (WOB), with HCPAP has a longer duration of positive pressure application due to patients improving tolerability. ${ }^{21,25}$ Surviving Sepsis Campaign did not specifically recommend these two interfaces option since their safety or efficacy in COVID-19 patients was uncertain. ${ }^{26}$ In essence, no interface is perfect; therefore interface selection requires careful evaluation of patient characteristics, mode of ventilation, and type of acute respiratory failure. The target of device selection is minimal air leakage, patient comfort, and optimization of patient-ventilator interaction. ${ }^{27}$ 


\section{Bilevel Positive Airway Pressure (BiPAP)}

While CPAP is used for hypoxemic respiratory failure, NIV BiPAP should be reserved for patients with hypercapnic one, either acute or chronic. It is usually not needed in patients with otherwise normal lungs since compliance is generally low or maintained in COVID-19 patients. In COVID-19 patients, it might be useful for those who have comorbidities such as COPD. The indications are similar to CPAP, such as (1) a ceiling for treatment, (2) a support to avoid intubation, and (3) facilitating extubation. ${ }^{20}$ The main difference with CPAP, BiPAP has two pressure settings, which are: the prescribed pressure during inspiration known as inspiratory positive airway pressure (IPAP) and a lower pressure for expiration known as expiratory positive airway pressure (EPAP). In CPAP, there is no additional supportive pressure above the set level. ${ }^{19}$ The patient must be assessed for a pneumothorax before BiPAP application since there is a risk of the excessive tidal volume received, resulting in barotrauma. ${ }^{6}$

Inspiratory pressure was pre-set to allow patients to breathe and achieve adequate $\mathrm{V}_{\mathrm{T}}$; thus, IPAP settings might be varied, ranging from 12 to $35 \mathrm{cmH}_{2} \mathrm{O}$. Meanwhile, the working principle of EPAP is the same as that of PEEP in CPAP machines, which can prevent the alveoli from collapsing during exhalation by maintaining the pressure above atmospheric pressure. Pressure support is also provided to help overcome breathing difficulties on the ventilator (including valves) and the increase in dead space caused by ventilator tube, and it is calculated by subtracting IPAP from EPAP. If oxygenation is required, it is recommended to have a difference of at least $8 \mathrm{cmH}_{2} \mathrm{O}^{6}$ from supplemental oxygen. Some BiPAP ventilators have a "ramp" setting, also called "rise time", which allows the pressure to be slowly increased during the first few minutes of ventilation until the desired pressure is reached. This prevents barotrauma and reduces the pressure on the patient at the beginning of treatment. This way, $25 \%$ of the rise time accounts for $25 \%$ of the total inspiration time before reaching the peak pressure. There is not much information regarding the use of BiPAP in COVID-19 patients, but it might still be useful, especially for those who have multiple comorbidities. ${ }^{24}$

\section{High-Flow Nasal Oxygen (HFNO)}

Compared to CPAP and BiPAP, HFNO is different in providing positive pressure to the airway. The continuous positive airway pressure was similar to CPAP but delivered through a nasal cannula, with higher flow up to 60 liters/min. HFNO has more advantages in providing warmed and humidified gases to prevent dry airway, and its high flows allow oxygen delivery almost $100 \%$, carbon dioxide 'washout', reduction of anatomical dead space by preventing rebreathing, and ensuring positive-end expiratory. The PEEP effect oxygenates the airway, and the warm water creates vapors that loosen mucus to improve mucus clearance, therefore the alveoli can fully expand. ${ }^{28}$ Subsequently, the reduction of anatomical dead space will enhance the work of breathing and lowering the respiratory rate. HFNO also reduce the metabolic demand of breathing, production of carbon dioxide, and provides greater comfort compared to mechanical ventilation ${ }^{29}$

CPAP is still considered the first choice for NIV in COVID-19 patients, partly because the evidence of HFNO in COVID-19 patients is still under debate. To be stated simply, the use of HFNO remains contentious with different perspectives on how this modality can be used to treat respiratory failure in COVID-19 patients. ${ }^{30}$ Surviving Sepsis Campaign (SSC) guidelines recommend HFNO over conventional oxygen therapy for COVID-19 patients with acute hypoxemic respiratory failure. ${ }^{26}$

For some physicians, the issue of aerosolization of virus in HFNO raises a lot of concern due to the high flow used, although some evidence found no increased risk of healthcare workers with HFNO, compared to intubation and mechanical ventilation. ${ }^{30}$ Therefore, due to its lack of evidence of efficacy, high oxygen consumption, and risk of infection transmission, some European guidelines currently do not recommend HFNO for COVID-19 patients. $^{20}$ These confusing choice regarding HFNO usage was also reflected in the first two COVID-19 clinical studies. One study in USA reported that only one severe COVID-19 patient out of 21 patients was given HFNO. In contrast, a study in Wuhan, China reported more than $50 \%$ patients in ICU used HFNO. ${ }^{30}$ World Health Organization (WHO) guideline supports the use of HFNO in some patients, but with close observation for rapid worsening that may result in intubation and increase the risk of medical workers getting infected. ${ }^{6}$ 
Some contraindications for HFNO including severe respiratory distress, uncooperative patients or patients with decreased of consciousness, facial injury, epistaxis, and airway obstruction. ${ }^{28}$ Although some literature mention the benefits of preventing intubation and mechanical ventilation in some critically ill patients with respiratory distress, ${ }^{31}$ the use of HFNO should be closely monitored because delayed intubation is associated with worse outcomes and it was said that early intubation may prevent the transition from $\mathrm{L}$ to $\mathrm{H}$ type. ${ }^{4}$ CPAP and NIV are also the main options for patients with respiratory distress to improve blood oxygenation. ${ }^{13}$ The use of high PEEP, in some patients, can reduce pressure swings in the pleura and stop the vicious cycle of exacerbated lung injury. However, a high PEEP in patients with normal compliance may have a detrimental effect on hemodynamics. In type L, PEEP should be limited to between $8-10 \mathrm{cmH}_{2} \mathrm{O}$ given the low recruitment and risk of hemodynamic failure increasing at higher rates. Whereas in type $\mathrm{H}$, a gradual increase in PEEP to $14-15 \mathrm{cmH}_{2} \mathrm{O}$ can provide benefits.

One thing to remember in using HFNO is that this device could dry up the airway lining due to its high flow system. Regular check on humidifier water chamber and fluid bag installed is necessary and important to maintain the water level inside and the temperature of $37^{\circ} \mathrm{C}$ for the system to work properly. HFNO usually starts with a flow rate of 60 liters/minute and titrated to reach the target saturation $\left(\mathrm{SpO}_{2}\right)$. If there is no improvement, the oxygen level should be increased until the target saturation is reached. However, if the oxygen content is $>50 \%$, the patient should be reviewed urgently because intubation might be necessary. In COVID-19 patients especially, the intubation team will need to enhance their personal protective equipment (PPE), which takes more time before possible intervention. When indicated, oxygen should be weaned first before the flow by decreasing it 5 liters at time, or as patient tolerated. ${ }^{6}$

\section{SUMMARY}

COVID-19 is a multisystem inflammatory complex with respiratory failure due to ARDS as the most frequent complication with a high mortality rate. CARDS has similar clinical signs, but with characteristics that are quite different from ARDS in general. ARDS should be understood as a syndrome or as a whole, whereas CARDS is a specific disease that causes ARDS. Understanding the clinical characteristics and proper pathophysiology of CARDS is a crucial point for early identification and determining the basis of appropriate management. Not only in terms of respiratory management but also other supportive therapies related to multi-organ damage that often occurs in COVID-19 patients.

The use of NIV, including HFNO, must pay attention to many aspects ranging from indication or patient selection, timing to start, understanding of predictor factors of failure, and selection of the right NIV interface. In principle, each patient has a different condition and should be treated case by case. NIV is not an absolute solution, intubation can still be the first choice if NIV is deemed less beneficial for the patient.

Both HFNO and NIV are controversial due to their use which increase aerosol transmission of the virus. Therefore, their usage for COVID-19 patients were highly recommended in negative pressure room. All the medical staffs caring for COVID-19 patients should protect themselves with proper PPE. A lot of trials have emerged to prove the efficacy of NIV and HFNO, but in fact the results are not all satisfying. There are some possibilities that they may be associated with high failure rates and delayed intubations, especially in disease that can usually last up to several weeks.

\section{Acknowledgments}

None declared.

\section{Conflict of Interest}

The author declared there is no conflict of interest.

\section{Funding}

This study did not receive any funding.

\section{Authors' Contributions}

Writing manuscript: IS Reviewing and revising: IS and YJC. All authors contributed and approved the final version of the manuscript.

\section{REFERENCES}

1. WHO Coronavirus Disease (COVID-19) Dashboard. Geneva: World Health Organization; 2021. Accessed September, 17th 2021. [WebPage]

2. Li X, Ma X. Acute Respiratory Failure in COVID19: Is It "typical" ARDS? Crit Care. 2020;24(1):198. [PubMed]

3. Wilcox SR. Management of Respiratory Failure due to COVID-19. BMJ. 2020;369:m1786. [PubMed]

4. Pfortmueller CA, Spinetti T, Urman RD, Luedi MM, Schefold JC. COVID-19 Associated Acute Respiratory Distress Syndrome (CARDS): Current Knowledge on Pathophysiology and ICU Treatment 
- A Narrative Review. Best Practice \& Research Clinical Anaesthesiology. 2020. [PubMed]

5. Scala R, Heunks L. Highlights in Acute Respiratory Failure. European Respiratory Review : An Official Journal of the European Respiratory Society. 2018;27(147):180008. [PubMed]

6. Carter C, Aedy H, Notter J. COVID-19 Disease: Non-Invasive Ventilation and High Frequency Nasal Oxygenation. Clinics in Integrated Care. 2020;1:100006-100006. [PubMed]

7. Gattinoni L, Chiumello D, Rossi S. COVID-19 Pneumonia: ARDS or Not? Crit Care. 2020;24(1):154. [PubMed]

8. Bellani G, Grasselli G, Cecconi $\mathrm{M}$, et al. Noninvasive Ventilatory Support of Patients with COVID-19 outside the Intensive Care Units (WARd-COVID). Annals of the American Thoracic Society. 2021;18(6):1020-1026. [PubMed]

9. Franco C, Facciolongo N, Tonelli R, et al. Feasibility and Clinical Impact of Out-of-ICU Noninvasive Respiratory Support in Patients with COVID-19 Related Pneumonia. The European Respiratory Journal. 2020;56(5). [PubMed]

10. Menzella F, Barbieri C, Fontana $\mathrm{M}$, et al. Effectiveness of Noninvasive Ventilation in COVID-19 Related-Acute Respiratory Distress Syndrome. Clin Respir J. 2021;15(7):779-787. [PubMed]

11. The ARDS Definition Task Force*. Acute Respiratory Distress Syndrome: The Berlin Definition. JAMA. 2012;307(23):2526-2533. [PubMed]

12. Gibson PG, Qin L, Puah SH. COVID-19 Acute Respiratory Distress Syndrome (ARDS): Clinical Features and Differences from Typical Pre-COVID19 ARDS. Med J Aust. 2020;213(2):54-56 e51. [PubMed]

13. Gattinoni L, Chiumello D, Caironi P, et al. COVID19 Pneumonia: Different Respiratory Treatments for Different Phenotypes? Intensive Care Medicine. 2020;46(6):1099-1102. [PubMed]

14. Scala R, Pisani L. Noninvasive Ventilation in Acute Respiratory Failure: Which Recipe for Success? European Respiratory Review : An Official Journal of the European Respiratory Society. 2018;27(149):180029. [PubMed]

15. Nava S, Welte T. European Respiratory Monograph 36: Respiratory Emergencies. European Respiratory Society Journals; 2006.

16. Nava S, Navalesi P, Conti G. Time of Non-Invasive Ventilation. Intensive Care Medicine. 2006;32(3):361-370. [PubMed]

17. Namendys-Silva SA. Respiratory Support for Patients with COVID-19 Infection. The Lancet. Respiratory Medicine. 2020;8(4):e18-e18. [PubMed]

18. ERS Practical Handbook of Noninvasive Ventilation. 2015.
19. Continuous Positive Airway Pressure. StatPearls Publishing; 2021. Accessed Updated 2021 Jul 31. [WebPage]

20. England N. Specialty Guides for Patient Management during the Coronavirus Pandemic. Guidance for the Role and Use of Non-Invasive Respiratory Support in Adult Patients with COVID19 (Confirmed or Suspected) 6 April 2020, Version 3. Version; 2020.

21. Amirfarzan H, Cereda M, Gaulton TG, et al. Use of Helmet CPAP in COVID-19 - A Practical Review. Pulmonology. 2021;27(5):413-422. [PubMed]

22. Tobin MJ. Basing Respiratory Management of COVID-19 on Physiological Principles. American Journal of Respiratory and Critical Care Medicine. 2020;201(11):1319-1320. [PubMed]

23. Vitacca M, Nava S, Santus P, Harari S. Early Consensus Management for Non-ICU Acute Respiratory Failure SARS-CoV-2 Emergency in Italy: From Ward to Trenches. The European Respiratory Journal. 2020;55(5). [PubMed]

24. Davies M, Allen M, Bentley A, et al. British Thoracic Society Quality Standards for Acute NonInvasive Ventilation in Adults. BMJ Open Respiratory Research. 2018;5(1):e000283. [PubMed]

25. Chiumello D, Pelosi P, Carlesso E, et al. Noninvasive Positive Pressure Ventilation Delivered by Helmet vs. Standard Face Mask. Intensive Care Medicine. 2003;29(10):1671-1679. [PubMed]

26. Alhazzani W, Møller MH, Arabi YM, et al. Surviving Sepsis Campaign: Guidelines on the Management of Critically Ill Adults with Coronavirus Disease 2019 (COVID-19). Intensive Care Medicine. 2020;46(5):854-887. [PubMed]

27. Nava S, Navalesi P, Gregoretti C. Interfaces and Humidification for Noninvasive Mechanical Ventilation. Respiratory Care. 2009;54(1):71-84. [PubMed]

28. Ashraf-Kashani N, Kumar R. High-Flow Nasal Oxygen Therapy. BJA Education. 2017;17(2):63-67. [WebPage]

29. Ballah A, Yusuf B, Ibrahim A, Dunga J, Zuwaira H, Maigari I. High Flow Nasal Oxygen Therapy in COVID-19 Patients; Our Experience in a Low Resource Setting. Nig Del Med. 2021:29-33. [WebPage]

30. Li J, Fink JB, Ehrmann S. High-Flow Nasal Cannula for COVID-19 Patients: Low Risk of Bio-Aerosol Dispersion. European Respiratory Journal. 2020;55(5):2000892. [PubMed]

31. Demoule A, Vieillard Baron A, Darmon M, et al. High-Flow Nasal Cannula in Critically III Patients with Severe COVID-19. American Journal of Respiratory and Critical Care Medicine. 2020;202(7):1039-1042. [PubMed] 